\title{
Effects of strong magnetic fields on hybrid stars
}

\author{
Bruno Franzon* \\ Frankfurt Institute for Advanced Studies, Ruth-Moufang - 1 60438, Frankfurt am Main, \\ Germany \\ E-mail: franzon@fias.uni-frankfurt.de \\ V. Dexheimer \\ Department of Physics, Kent State University, Kent OH 44242 USA \\ E-mail: vdexheim@kent.edu

\section{S. Schramm} \\ Frankfurt Institute for Advanced Studies, Ruth-Moufang - 1 60438, Frankfurt am Main, \\ Germany \\ E-mail: schramm@fias.uni-frankfurt.de
}

In this work we study the effects of strong magnetic fields on hybrid stars by using a full general-relativity approach, solving the coupled Maxwell-Einstein equation in a selfconsistent way. The magnetic field is assumed to be axi-symmetric and poloidal. We take into consideration the anisotropy of the energy-momentum tensor due to the magnetic field, magnetic field effects on equation of state, the interaction between matter and the magnetic field (magnetization), and the anomalous magnetic moment of the hadrons. The equation of state used is an extended hadronic and quark $\mathrm{SU}(3)$ non-linear realization of the sigma model that describes magnetized hybrid stars containing nucleons, hyperons and quarks. According to our results, the effects of the magnetization and the magnetic field on the EoS do not play an important role on global properties of these stars. On the other hand, the magnetic field causes the central density in these objects to be reduced, inducing major changes in the populated degrees of freedom and, potentially, converting a hybrid star into a hadronic star.

The Modern Physics of Compact Stars 2015

30 September 2015 - 3 October 2015

Yerevan, Armenia

*Speaker. 


\section{Introduction}

Neutron stars undoubtedly belong to the most suitable environments for studying properties of strongly interacting matter under extreme conditions. For example, the density inside these objects can reach values much higher than the nuclear saturation density $\sim 2.7 \times 10^{14} \mathrm{~g} / \mathrm{cm}^{3}$. This makes neutron stars natural laboratories where one can examine and shed some light on the still open question concerning the equation of state (EoS) for ultra-dense matter and the role played by exotic degrees of freedom, such as hyperons and quarks.

Another important feature present in compact objects and studied in this work is their strong magnetic field. From pulsar observations, the magnitude of the surface magnetic field in neutron stars has been found to be generally of the order of $10^{12}-10^{13} \mathrm{G}$. However, according to observations of star periods and period derivatives, classes of neutron stars known as Anomalous X-ray Pulsars and Soft-Gamma-Ray-Repeaters can have surface magnetic field as large as $10^{14}-10^{15} \mathrm{G}$. These are usually referred to as magnetars, see e.g. $[1,2,3,4,5]$. One expects to find even stronger magnetic fields inside these stars as already calculated in [6]. According to virial theorem arguments, which give an upper estimate for the magnetic inside neutron stars, they can possess central magnetic fields as large as $10^{18-20} \mathrm{G}$, see e.g. [7, 8, 9, 10].

The origin of strong magnetic fields in compact stars is still unclear. One common hypothesis involves the flux conservation of the progenitor magnetic field, see [11]. However, this idea is not suitable for magnetars since a canonical neutron star $\mathrm{M} \sim 1.4 \mathrm{M}_{\odot}$ would require a radius less than its Schwarzschild radius in order to generate a surface magnetic field of the order of $10^{15} \mathrm{G}$, see [12]. Another possibility suggested by Thompson and Duncan in [1] describes a newly born neutron star combining convection and differential rotation to generate a dynamo process which is able to generate fields as large as $10^{15} \mathrm{G}$. However, this standard explanation fails when trying to explain the supernova remnants associated with these objects, see e.g. [13].

Whatever the origin of strong magnetic fields might be, they affect locally the microphysics of the equation of state (EoS), as for example, due to the Landau quantization of the energy levels of charged particles and the effect of the anomalous magnetic moment (AMM) of charged and uncharged particles. Globally, magnetic fields affect the structure of neutron stars through the Lorentz force associated with the macroscopic currents that generate the field. They also affect the structure of the spacetime, as magnetic fields are now a source for the gravitational field through the Maxwell energy-momentum tensor. As a consequence, magnetized stars are anisotropic and require a general-relativity treatment beyond the solution of the Tolman-Oppenheimer-Volkoff (TOV) equations ([14, 15]).

In this work, we model magnetized hybrid stars in a self-consistent way by solving Einstein-Maxwell equations in the same way as done in [16, 17]. In [17], the authors studied magnetized quark stars taking into account the magnetic field in the equation of state, the magnetization term for the matter, and also the magnetic field in the gravitational field equations. They have found that neither the magnetic field nor the magnetization change significantly the global properties of these stars for a magnetic field strength of the order 
of $\sim 10^{18} \mathrm{G}$. Note that, the equation of state used by those authors, namely, a quark CFL model ([18]) possesses a high baryon density range reaching two times the saturation density at the surface of star.

In order to assess the role that a magnetic field dependent equation of state and the magnetization play in the global properties of stars, we use in this work a more complex equation of state than in [17]. Our EoS describes magnetized hybrid stars containing nucleons, hyperons and quarks and takes into account the anomalous magnetic moment for all hadrons. As a consequence, it produces a magnetization much higher than the one used in [17]. Despite this, we show that the neutron star structure, like its mass-radius relationship, is not modified drastically with the inclusion of the magnetic field in the EoS and the magnetization. On the other hand, the particle population is significantly modified when the magnetic field is included. The main impact observed is the conversion of a nonmagnetized hybrid star with hadron and quark degrees of freedom to a highly magnetized hadronic star composed simply by nucleons. In reality, the temporal star evolution goes in the other direction, as the magnetic field of the star decays over time allowing a hadronic star to become a hybrid one.

\section{Formalism}

We present here the effects of strong magnetic fields on the global properties of neutron stars which are subjected to a quark-hadron phase transition in their interior. Stationary and axi-symmetric stellar models were constructed with the same numerical procedure and mathematical set-up as in Refs. [19, 17], where the coupled Maxwell-Einstein equations were solved in a self-consistent way by means of a pseudo-spectral method. The magnetic models are obtained by giving a constant current functions $f_{0}$, which is related to the macroscopic electric current through the relation $j^{\phi} \propto(e+p) f_{0}$, where $e$ and $p$ are the energy density and the pressure of the system.

\section{Magnetized Equation of State}

When, in addition to a usual hadronic phase, neutron star models also include a quark phase, they are normally described by two different equations of state. These equations of state are connected at the point in which the pressure of the quark phase becomes higher than the pressure of the hadronic phase. In our approach, we have instead a combined model of hadrons and quarks and one equation of state for both phases. In this case, we can study important features of the deconfinement phase transition, like the strength of the transition, the mixing of phases and also the accompanying chiral symmetry restoration. For this purpose, we extended the hadronic SU(3) non-linear realization of the sigma model, see e.g. [20, 21, 22, 23], to include quark degrees of freedom in a spirit similar to the PNJL model ([24]), in the sense that it uses the Polyakov loop $\Phi$ as the order parameter for deconfinement. The Lagrangian density of the model in mean field approximation reads:

$$
\mathscr{L}=\mathscr{L}_{\text {mag }}+\mathscr{L}_{\text {Kin }}+\mathscr{L}_{\text {Int }}+\mathscr{L}_{\text {Self }}+\mathscr{L}_{S B}-U
$$


where besides the kinetic energy term for hadrons, quarks, and leptons (included to insure charge neutrality), the terms:

$$
\begin{gathered}
\mathscr{L}_{\text {mag }}=-\sum_{i} \bar{\psi}_{i}\left(q_{i} e \gamma^{\mu} A_{\mu}+\frac{1}{2} \kappa \sigma^{\mu v} F_{\mu v}\right) \psi_{i} \\
\mathscr{L}_{\text {Int }}=-\sum_{i} \bar{\psi}_{i}\left[\gamma_{0}\left(g_{i \omega} \omega+g_{i \phi} \phi+g_{i \rho} \tau_{3} \rho\right)+M_{i}^{*}\right] \psi_{i} \\
\mathscr{L}_{\text {Self }}=\frac{1}{2}\left(m_{\omega}^{2} \omega^{2}+m_{\rho}^{2} \rho^{2}+m_{\phi}^{2} \phi^{2}\right)+g_{4}\left(\omega^{4}+\frac{\phi^{4}}{4}+3 \omega^{2} \phi^{2}+\frac{4 \omega^{3} \phi}{\sqrt{2}}+\frac{2 \omega \phi^{3}}{\sqrt{2}}\right)-k_{0}\left(\sigma^{2}+\zeta^{2}+\delta^{2}\right) \\
-k_{1}\left(\sigma^{2}+\zeta^{2}+\delta^{2}\right)^{2}-k_{2}\left(\frac{\sigma^{4}}{2}+\frac{\delta^{4}}{2}+3 \sigma^{2} \delta^{2}+\zeta^{4}\right)-k_{3}\left(\sigma^{2}-\delta^{2}\right) \zeta \\
-k_{4} \ln \frac{\left(\sigma^{2}-\delta^{2}\right) \zeta}{\sigma_{0}^{2} \zeta_{0}} \\
\mathscr{L}_{S B}=-m_{\pi}^{2} f_{\pi} \sigma-\left(\sqrt{2} m_{k}^{2} f_{k}-\frac{1}{\sqrt{2}} m_{\pi}^{2} f_{\pi}\right) \zeta \\
U=\left(a_{0} T^{4}+a_{1} \mu^{4}+a_{2} T^{2} \mu^{2}\right) \Phi^{2}+a_{3} T_{0}^{4} \log \left(1-6 \Phi^{2}+8 \Phi^{3}-3 \Phi^{4}\right)
\end{gathered}
$$

represent the magnetic and anomalous magnetic moment (AMM) interactions with the fermions, the interactions between baryons or quarks and vector and scalar mesons, the self interactions of scalar and vector mesons, an explicit chiral symmetry breaking term (responsible for producing the masses of the pseudo-scalar mesons), and a potential $U$ for the $\Phi$ field. The later is important in order to reproduce a realistic structure for the QCD phase diagram over the whole range of chemical potentials and temperatures, including realistic thermodynamic behaviour at vanishing chemical potential as shown in [22].

The effective masses of the baryons and quarks are generated by the scalar mesons except for a small explicit mass term $M_{0}$ and the term containing $\Phi$ :

$$
\begin{gathered}
M_{B}^{*}=g_{B \sigma} \sigma+g_{B \delta} \tau_{3} \delta+g_{B \zeta} \zeta+M_{0_{B}}+g_{B \Phi} \Phi^{2} \\
M_{q}^{*}=g_{q \sigma} \sigma+g_{q \delta} \tau_{3} \delta+g_{q \zeta} \zeta+M_{0_{q}}+g_{q \Phi}(1-\Phi) .
\end{gathered}
$$

With the increase of temperature and/or density, the $\sigma$ field (non-strange chiral condensate) decreases in value, causing the effective masses of the particles to decrease towards chiral symmetry restoration. The field $\Phi$ assumes non-zero values with the increase of temperature/density and, due to its presence in the baryons effective mass (Eq. (3.7)), suppresses their presence. On the other hand, the presence of the $\Phi$ field in the effective mass of the quarks, included with a negative sign (Eq. (3.8)), ensures that they will not be present at low temperatures/densities. In this way, the interaction with the medium determines which are the degrees of freedom present in the system.

The magnetic field in the z-direction forces the energy eigenstates in the $\mathrm{x}$ and $\mathrm{y}$ directions of the charged particles to be quantized into Landau levels $v$ :

$$
E_{i_{v s}}^{*}=\sqrt{k_{z_{i}}^{2}+\left(\sqrt{M_{i}^{* 2}+2 v\left|q_{i}\right| B}-s_{i} \kappa_{i} B\right)^{2}},
$$


where $k_{i}$ is the fermi momentum and $s_{i}$ the spin of each fermion. The last term comes from the anomalous magnetic moment (AMM) of the particle that splits the energy levels with respect to the alignment/anti-alignment of the spin with the magnetic field. The AMM also modifies the energy levels of the uncharged particles

$$
E_{i_{s}}^{*}=\sqrt{k_{i}^{2}+\left(M_{i}^{* 2}-s_{i} \kappa_{i} B^{*}\right)^{2}} .
$$

The AMM constants $\kappa_{i}$ have values $\kappa_{p}=1.79, \kappa_{n}=-1.91, \kappa_{\Lambda}=-0.61, \kappa_{\Sigma}^{+}=1.67, \kappa_{\Sigma}^{0}=1.61$, $\kappa_{\Sigma}^{-}=-0.38, \kappa_{\Xi}^{0}=-1.25, \kappa_{\Xi}^{-}=0.06$. The sign of $\kappa_{i}$ determines the preferred orientation of the spin with the magnetic field. For zero temperature, the sum over the Landau levels $v$ runs up to a maximum value, beyond which the momentum of the particles in the $\mathrm{z}-$ direction would be imaginary

$$
v_{\max }=\frac{E_{i_{s}}^{* 2}+s_{i} \kappa_{i} B-M_{i}^{* 2}}{2\left|q_{i}\right| B} .
$$

We choose to include in our calculations the AMM effect for the hadrons only, since the coupling strength of the particles $\kappa_{i}$ depends on the corresponding magnetic moment, that up to now is not fully understood for the quarks. Furthermore, it is stated in [25], that quarks in the constituent quark model have no anomalous magnetic moment, and in [26], that the AMM of quarks from one-loop fermion self-energy is very small. For calculations including AMM effects for the quarks, see [27, 28, 29, 30]. The AMM for the electrons is also not taken into account as its effect is negligibly small. Properties of the magnetized $\mathrm{SU}(3)$ non-linear realization of the sigma model were presented in [31,32] for an effective (ad hoc) variation of the magnetic field inside the star.

\section{Results}

The equilibrium configurations are determined by the central enthalpy $H_{c}$ and the choice of the magnetic dipole moment $\mu$. We could have, instead, chosen a fixed current function $f_{0}$ and allow the magnetic dipole moment to vary. We chose the former in order to have a better control of the parameter space and to investigate exclusively the effect of the magnetic field on the star. In order to do so, we built equilibrium sequences for fixed magnetic dipole moments $\mu$ defined as (see [16]):

$$
\frac{2 \mu \cos \theta}{r^{3}}=\left.B_{(r)}\right|_{r \rightarrow \infty}
$$

which is simply the radial component (the orthonormal one) of the magnetic field of a magnetic dipole seen by an observer at infinity.

The magnetization is defined as $M=-\partial \Omega / \partial B$ (for more details see [33]) and in Figure 1 we show: i) the case when the magnetic field is included only in the structure of the star (no EoS(B), no mag); ii) the effect of the magnetic field also into the equation of state on the neutron star structure without the magnetization term (EoS(B), no mag) and iii) the effect of the magnetic field also into the equation of state on the neutron star structure plus the 


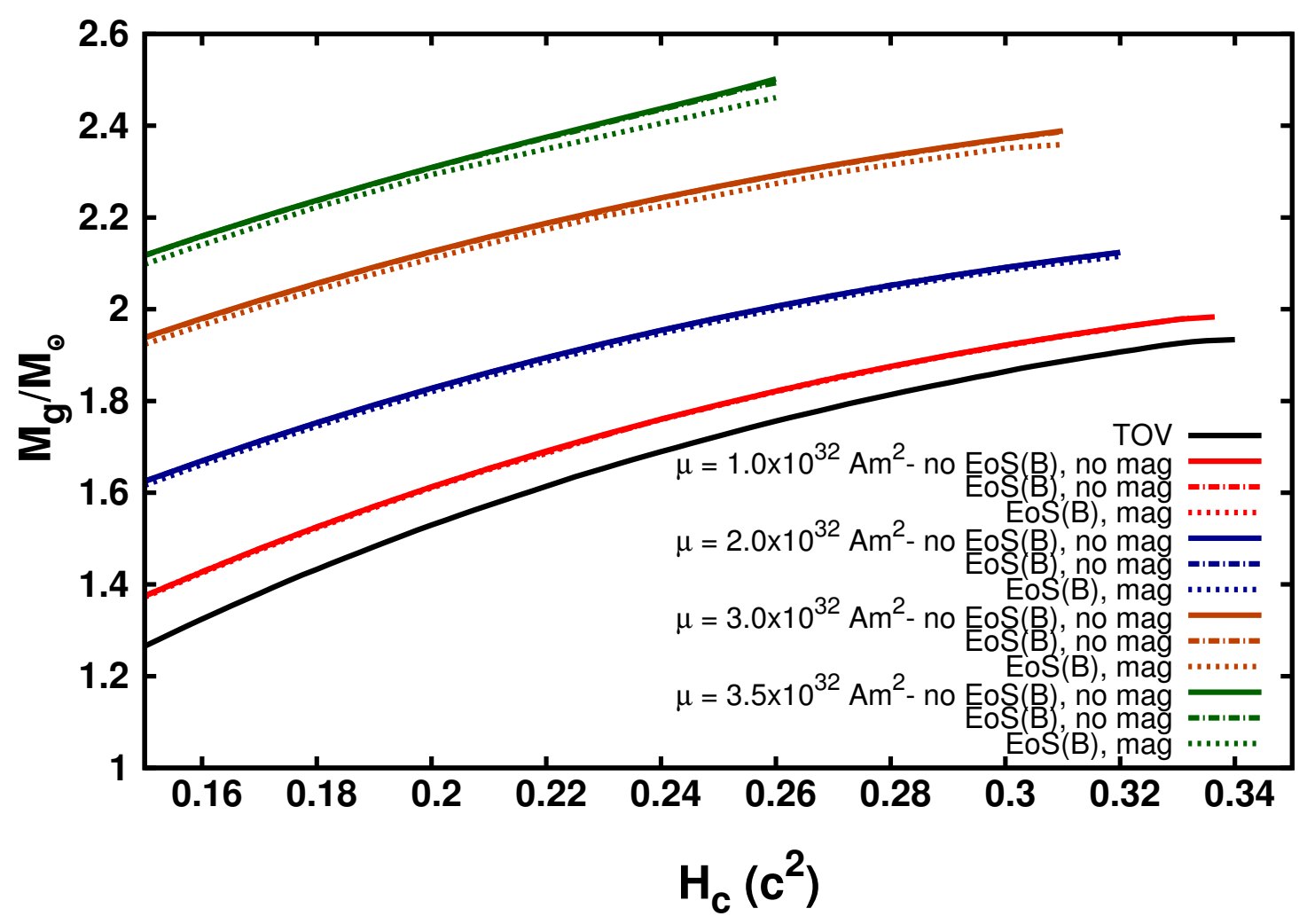

Figure 1: Relation between the gravitational mass and the central enthalpy for non-magnetized and magnetized models. In the last case, we also include the effects of the magnetic field into the equation of state $(\operatorname{EoS}(\mathrm{B}))$ and the magnetization term (mag) .

magnetization term (EoS(B), mag). We also show the non-magnetized cased denominated TOV.

The results present in [17] show that the effects of the magnetic field in the equation of state and the magnetization on the neutron star structure are not considerable. Note, however, that those authors presented solutions for magnetized quark stars whose equation of state (CFL model) is very stiff and reaches, for instance, a number baryon density of about 2 times the nuclear saturation density at the surface of the star. In this work, we model not only the quark phase with up, down quarks, but also the hadronic phase containing the whole baryon octet, in a self-consistent way in the presence of the magnetic field.

The results in Figure 1 corroborate in part the results presented in [17], showing also that the magnetic field does not have a considerable effect on the maximum mass and radius of highly magnetized neutron stars through the effect on the equation of state for given magnetic fields. Only a very small reduction of the star mass is seen for the largest magnetic moment used in the most massive star calculated. We see, however, from our calculation a difference in the curves when the magnetization term is included. This is due 
to the fact that, in our case, the magnetization strength can reach a value of about 10 times the value as in [17]. Note that the effect of the magnetization is to decrease stellar masses.

From this point on, all the results shown will include, for consistency, the magnetic field effect in the EoS and the magnetization effect. The mass-radius diagram for highly magnetized neutron stars determined by a constant magnetic dipole moment $\mu$ is presented in Figure 2. In this figure, we also show calculations for evolutionary sequences at fixed star baryonic mass of $\mathrm{M}_{\mathrm{B}}=2.2 \mathrm{M}_{\odot}$. These lines may represent the transition from a highly magnetized neutron star (a younger star) to a non-magnetized one (an older star).

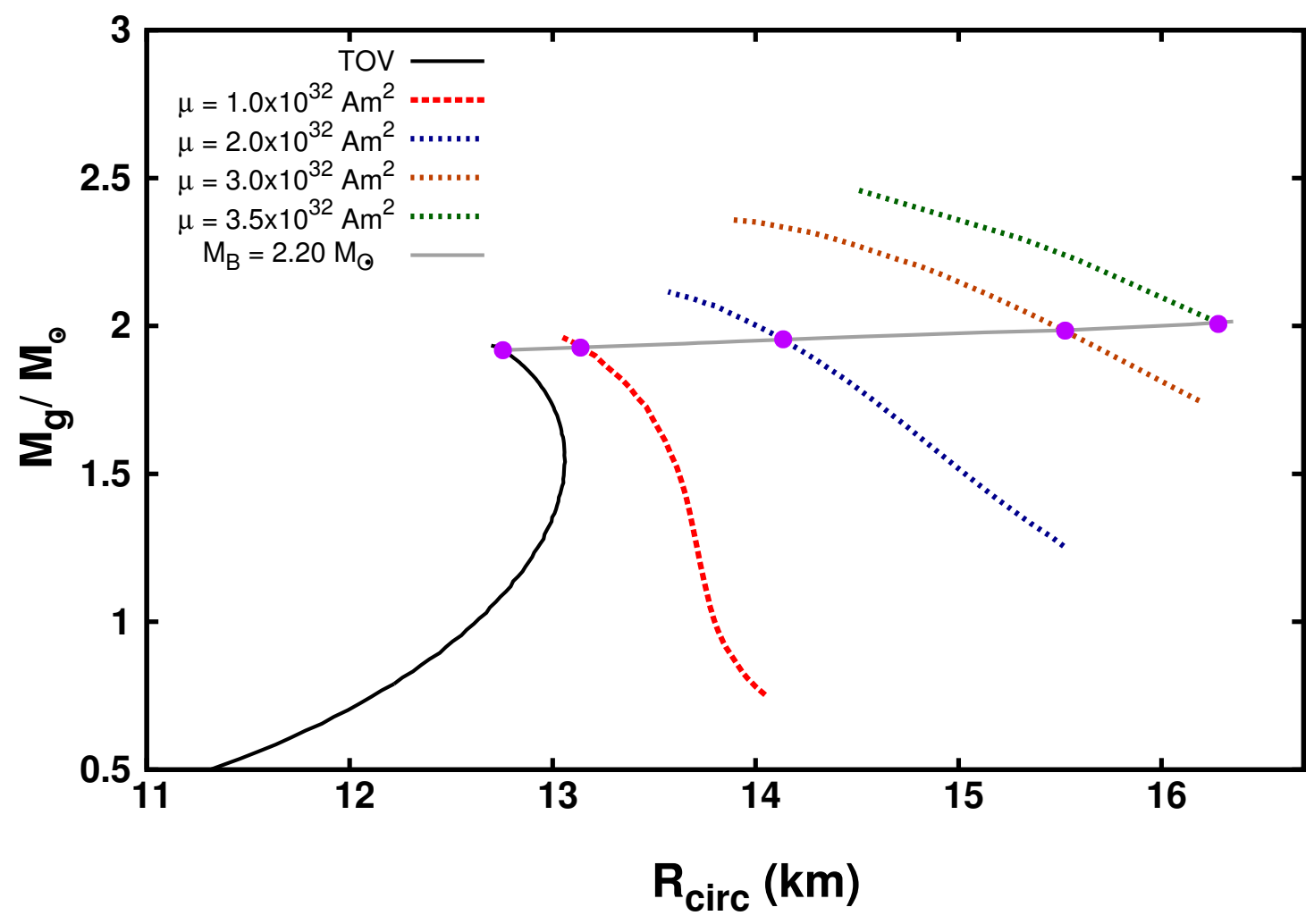

Figure 2: Mass-radius diagram for non-magnetized and magnetized models. The calculation was done for different fixed magnetic moments $\mu$. The higher the magnetic moment, the higher the magnetic field. Effects of the magnetic field into the equation of state and the magnetization are also included. The gray line shows an equilibrium sequence for a fixed baryon mass of $2.2 \mathrm{M}_{\odot}$. The full purple circles represent a possible evolution from a highly magnetized neutron star to a non-magnetized and spherical star.

We have chosen a fixed baryon mass of $2.2 \mathrm{M}_{\odot}$ because its evolution line ends almost at the maximum mass for the non-magnetized and spherical configuration. Looking at different magnetic dipole moment lines, i.e $\mu=1.0 \times 10^{32} \mathrm{Am}^{2}, 2.0 \times 10^{32} \mathrm{Am}^{2}, \mu=3.0 \times$ $10^{32} \mathrm{Am}^{2}$ and $\mu=3.5 \times 10^{32} \mathrm{Am}^{2}$, one sees that increasing $\mu$ (and therefore the magnetic field) affects the structure of the neutron star in many ways. First, the maximum mass increases, but not so much as in the case of no-fixed baryon number, what had been already 
raised in [34] using spherical approach. This is an effect of the Lorentz force acting outward and against gravity. For this reason, the star can support more mass. Second, the circular equatorial radius of the sequence increases and the star becomes much more deformed with respect to the symmetry axis. This deformation is also an effect of the assumption of a poloidal magnetic field, which makes the star more oblate. Calculations including toroidal magnetic field components have shown that magnetized stars become more prolate with respect to the non-magnetized case, see e.g. [35, 36, 37].

Figure 3 shows the magnetic field profile and the enthalpy iso-contours for a star with the maximum mass for a magnetization $\mu=3.5 \times 10^{32} \mathrm{Am}^{2}$, as can be seen in Figure 2 . This value roughly corresponds to the solution with maximum field configuration achieved with the code.

Now we follow the fixed baryon mass $\mathrm{M}_{\mathrm{B}}=2.2 \mathrm{M}_{\odot}$ in Figure 2 and present microscopic and macroscopic properties as a function of the central magnetic field in the stars. First, as shown in Fig. 4, the central baryon number density decreases with the central magnetic field and it has the maximum value at the center only in the static case. In the other cases, its maximum is found somewhere inside the star. This is due to the Lorentz force, which is related to the macroscopic currents that create the magnetic field, acting on the matter which has been pushed off-center. This is analogous to the number density reduction in the rotating star case. As we will see, this has a huge impact on the particle population of these objects. Second, the gravitational mass also increases, as already mentioned in this section. Third, as we fixed the baryon mass $M_{B}$, each star in the sequence reproduces different values of the central enthalpy $H_{c}$ and a different magnetic dipole moment $\mu$. The latter is free to vary and increases with the central magnetic field. Fourth, the ratio between the polar and the equatorial radii increases as the magnetic field increases and, therefore, the star becomes more deformed (oblate). For all quantities in Figure 4, the curves have a qualitatively change in behaviour for a magnetic field strength of $0.9-1.0 \times 10^{18} \mathrm{G}$. At this point, the magnetic force has pushed the matter off-center and a topological change to a toroidal configuration can take place ([10]). However, our current numerical tools do not enable us to handle toroidal configuration, which gives a limit for the magnetic field strength that we can obtain within this approach. As a consequence, the baryon number density, for example, will never reach zero at the center of the star. Still, the value of the magnetic field shown in Figure 4 represents the limit in terms of magnetic field strength for a star at fixed baryon mass of $\mathrm{M}_{\mathrm{B}}=2.2 \mathrm{M}_{\odot}$. Other configurations, as depicted in Figure 3, can reach values higher than $1.0 \times 10^{18} \mathrm{G}$.

Note that, in Figure 4, the ratio between the polar and the equatorial radii can reach $50 \%$ for a magnetic field strength of $\sim 1 \times 10^{18} \mathrm{G}$ at the center. Therefore, one sees that the deviations from spherical symmetry are quite significant and need to be taken into consideration while modelling these highly magnetized objects and a simple TOV solution can not be applied.

The changes in the global properties of stars due to the inclusion of the magnetic field into the gravitational equations are remarkable, and in order to study how the microphysics is modified with the magnetic field, we present in Figure 5 the particle population $Y_{i}=\rho_{i} / \rho_{b}$ as a function of the baryon chemical potential $\mu_{B}$ for different values of the magnetic dipole 
magnetic field
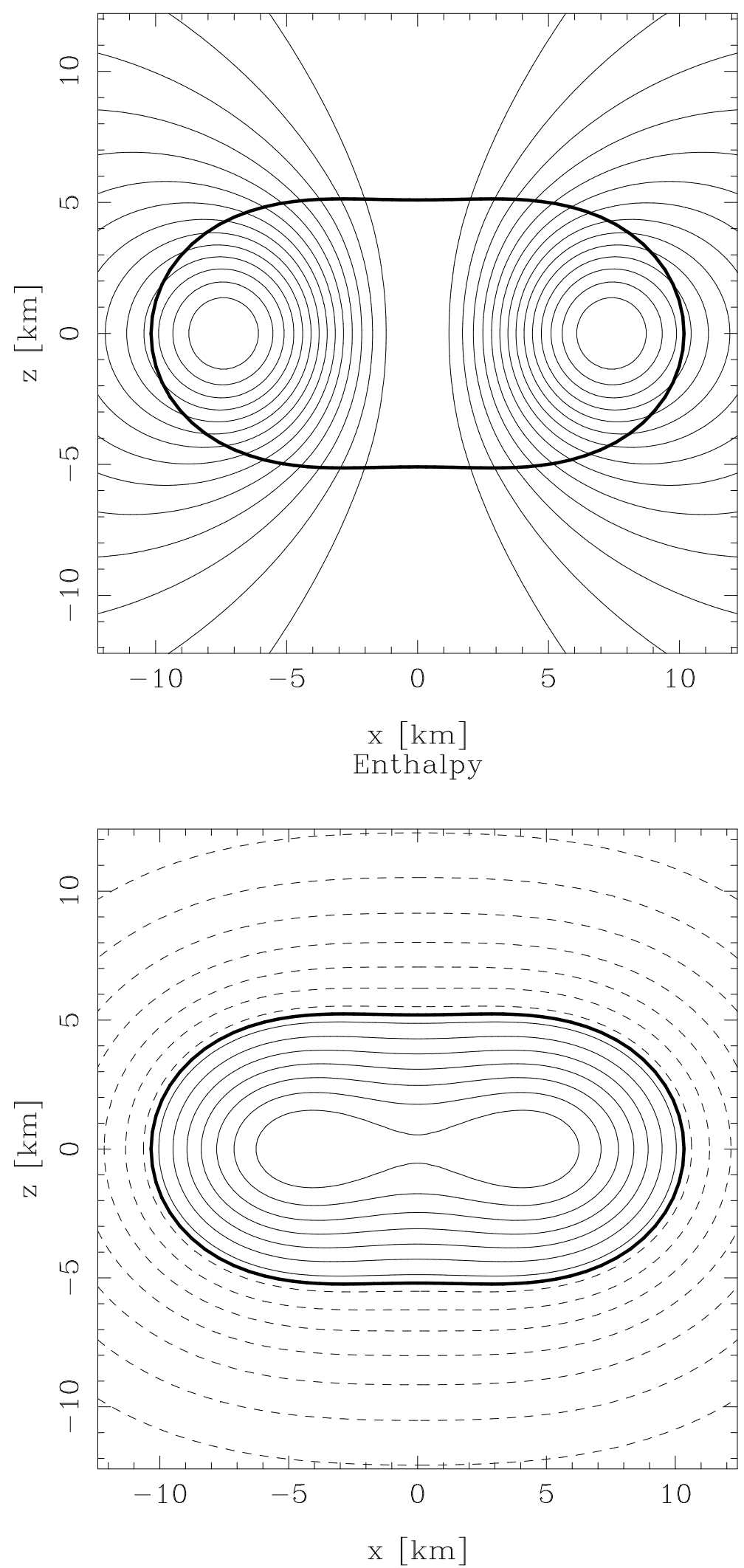

Figure 3: Magnetic field surfaces (on the top panel), i.e $A_{\phi}$ iso-contours measured by the Eulerian observer $\mathscr{O}_{0}$ for the chiral EoS. This star is near the maximum equilibrium configuration achieved by the code and the maximum mass for the value $\mu=3.5 \times 10^{32} \mathrm{Am}^{2}$, as shown in Figure 3 . In the bottom panel, the corresponding enthalpy profile is shown, which corresponds to a central enthalpy of $H_{c}=0.26 c^{2}\left(n=0.463 \mathrm{fm}^{-3}\right)$. The gravitational mass obtained for the star is $2.46 \mathrm{M}_{\odot}$ and the polar and the central magnetic fields are $8.59 \times 10^{17} \mathrm{G}$ and $1.62 \times 10^{18} \mathrm{G}$, respectively. The ratio between the magnetic pressure and the matter pressure in the center for this star is 0.793 . 

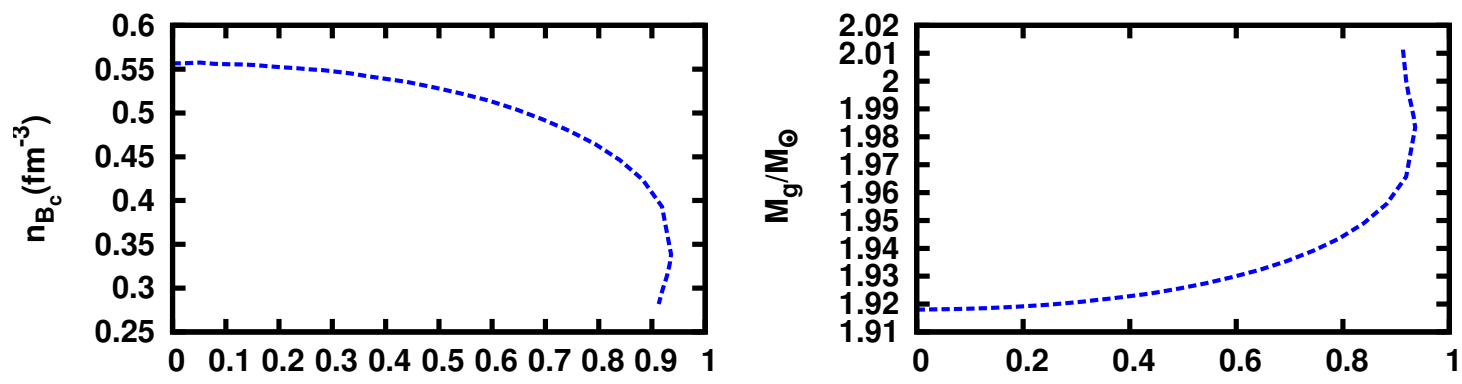

$B_{c}\left(10^{18} G\right)$
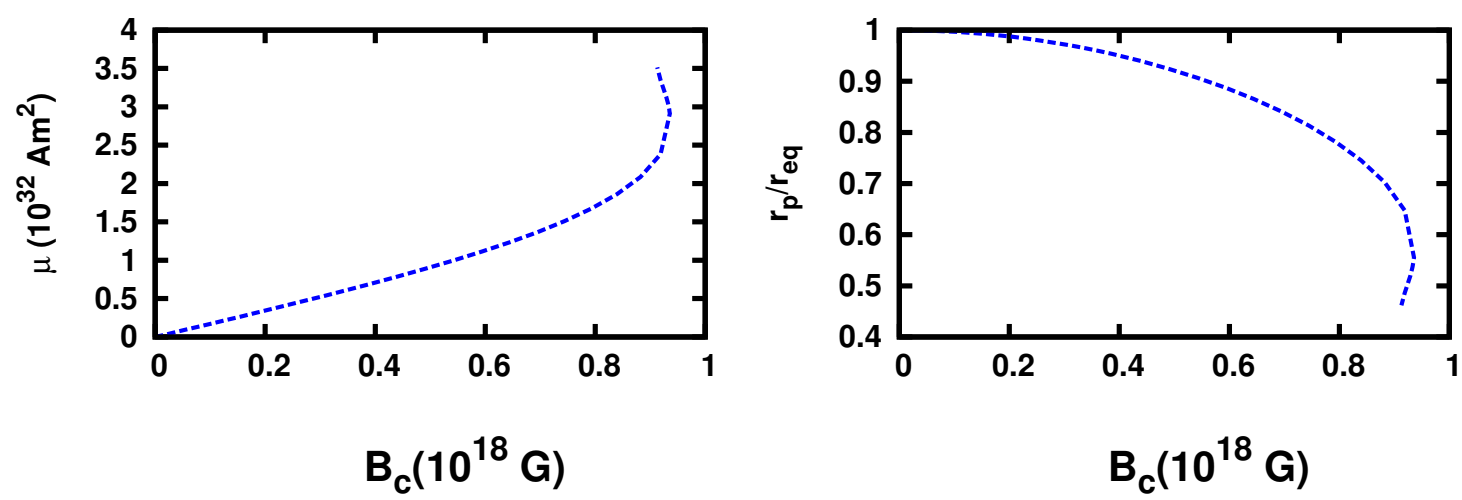

Figure 4: Microscopic and macroscopic star quantities, i.e central baryon number, gravitational mass, dipole magnetic moment and ratio between polar and equatorial coordinate radii, as a function of the central magnetic field. These curves represent an equilibrium sequence at fixed baryon mass $\mathrm{M}_{\mathrm{B}}=2.2 \mathrm{M}_{\odot}$ for different magnetic field intensities. See the line for $\mathrm{M}_{\mathrm{B}}=2.2 \mathrm{M}_{\odot}$ in Fig. 3).

moment $\mu$ for a fixed baryon mass $\mathrm{M}_{\mathrm{B}}=2.2 \mathrm{M}_{\odot}$. The kinks in the population plot that can be observed for values equal to or greater than $\mu=2.0 \times 10^{32} \mathrm{Am}^{2}$ are due to the Landau quantization.

For the spherical non-magnetized case, the TOV solution was obtained for a hybrid star (with mixed phase) composed by the baryon octet, electrons, muons and $\mathrm{u}, \mathrm{d}$ and $\mathrm{s}$ quarks. In Figure 5, the red vertical line represents the baryon chemical potential reached at the center of the maximum mass star in the non-magnetized case. With the inclusion of the magnetic field through the dipole magnetic moment of $\mu=1.0 \times 10^{32} \mathrm{Am}^{2}$, the central baryon chemical potential is reduced due to the Lorentz force. The new central value for $\mu_{B}$ is below threshold for the creation of quarks, which are, therefore, suppressed. An even larger effect can be seen in the star for higher values of the magnetic dipole moment $\mu=2.0 \times 10^{32} \mathrm{Am}^{2}, \mu=3.5 \times 10^{32} \mathrm{Am}^{2}$, when even the hyperons are suppressed. As a result, the properties of these objects such as neutrino emission and consequently the star cooling, are strongly affected by the magnetic field strength in their interior as already pointed out in [31] for a spherical solution.

In this way, younger stars that possess strong magnetic fields might go through a phase 


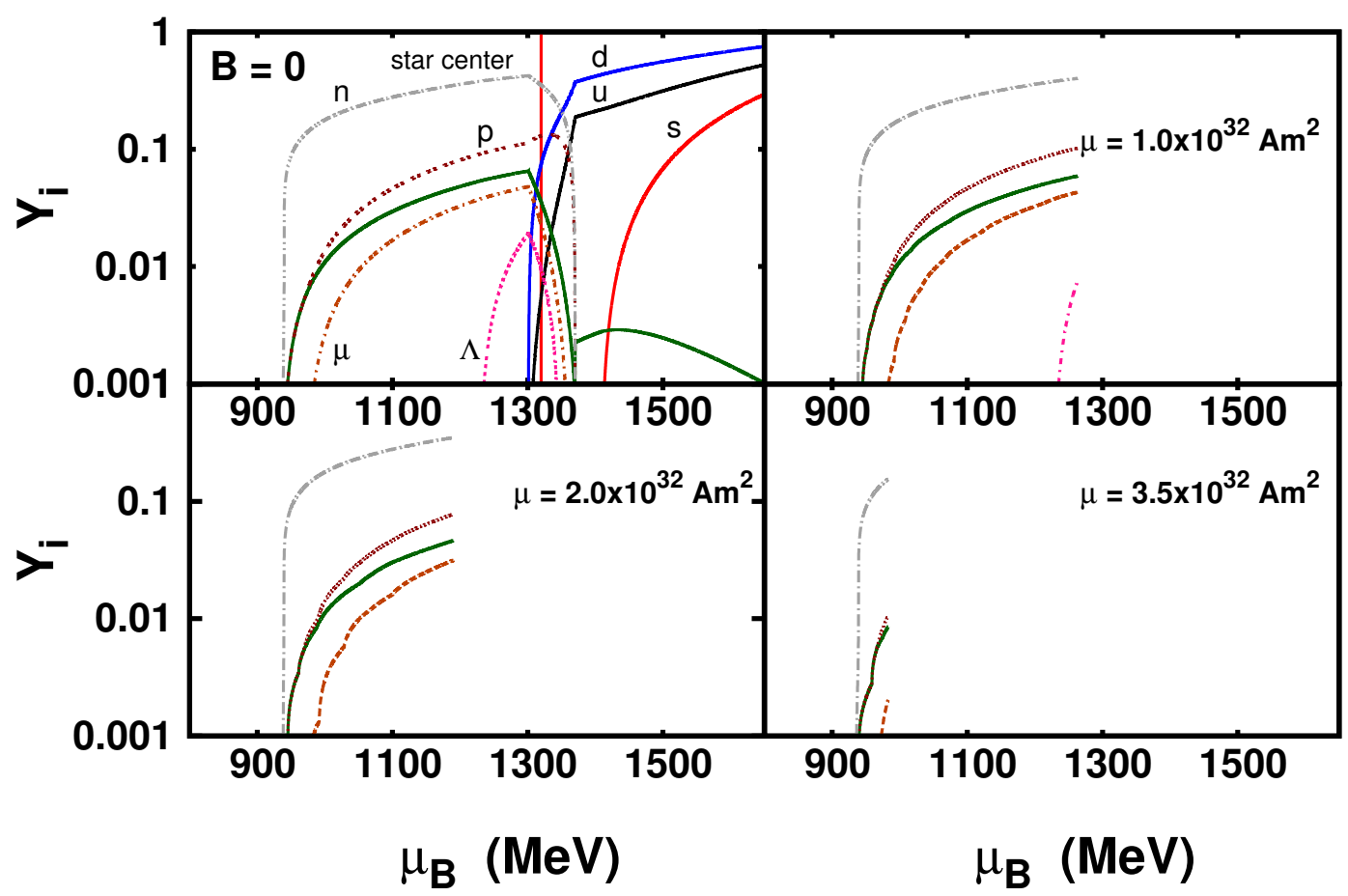

Figure 5: Stellar particle population as a function of the baryon chemical potential. All figures represent an equilibrium sequence at fixed baryon mass $\mathrm{M}_{\mathrm{B}}=2.2 \mathrm{M}_{\odot}$. As one increases the magnetic field, the particle population changes inside the star. These stars are represented in Fig. 2 by the full purple circles. For the non-magnetized case $(B=0)$, the vertical red line represents the chemical potential reached at the center of the star, namely, $1320 \mathrm{MeV}$.

transition later along their evolution, when their central densities increase enough for the hyperons and quarks to appear. Such phenomena might have observable signatures such as a distinct change in the cooling behaviour as well as a very different rotational slowing down, reflected in the stellar braking index.

\section{Acknowledgement}

We thank M. Strickland for fruitful comments and Joachim Frieben and D. Chatterjee for the valuable help with the numerical code. B. Franzon acknowledges support from CNPq/Brazil, DAAD and HGS-HIRe for FAIR. S. Schramm acknowledges support from the HIC for FAIR LOEWE program. The authors wish to acknowledge the "NewCompStar" COST Action MP1304.

\section{References}

[1] R. C. Duncan and C. Thompson, Formation of very strongly magnetized neutron stars implications for gamma-ray bursts, Astrophys. J. 392 (1992) L9. 
[2] C. Thompson and R. C. Duncan, Neutron star dynamos and the origins of pulsar magnetism, Astrophys. J. 408 (1993) 194.

[3] C. Thompson and R. C. Duncan, The Soft gamma repeaters as very strongly magnetized neutron stars. 2. Quiescent neutrino, x-ray, and Alfven wave emission, Astrophys. J. 473 (1996) 322 .

[4] B. Paczynski, Gb 790305 as a very strongly magnetized neutron star, Acta Astronomica 42 (1992) 145-153.

[5] A. Melatos, Bumpy spin-down of anomalous x-ray pulsars: The link with magnetars, The Astrophysical Journal Letters 519 (1999) L77.

[6] K. Makishima, T. Enoto, J. S. Hiraga, T. Nakano, K. Nakazawa, S. Sakurai et al., Possible Evidence for Free Precession of a Strongly Magnetized Neutron Star in the Magnetar $4 U$ 0142+61, Phys. Rev. Lett. 112 (2014) 171102.

[7] E. J. Ferrer, V. de La Incera, J. P. Keith, I. Portillo and P. L. Springsteen, Equation of state of a dense and magnetized fermion system, Physical Review C $\mathbf{8 2}$ (2010) 065802.

[8] D. Lai and S. L. Shapiro, Cold equation of state in a strong magnetic field-effects of inverse beta-decay, The Astrophysical Journal 383 (1991) 745-751.

[9] I. Fushiki, E. Gudmundsson and C. Pethick, Surface structure of neutron stars with high magnetic fields, The Astrophysical Journal 342 (1989) 958-975.

[10] C. Y. Cardall, M. Prakash and J. M. Lattimer, Effects of strong magnetic fields on neutron star structure, The Astrophysical Journal 554 (2001) 322.

[11] W. L.The Astrophysical Journal 140 (1964) 1309.

[12] T. Tatsumi, Ferromagnetism of quark liquid, Physics Letters B 489 (2000) 280-286.

[13] J. Vink and L. Kuiper, Supernova remnant energetics and magnetars: no evidence in favour of millisecond proto-neutron stars, Monthly Notices of the Royal Astronomical Society: Letters 370 (2006) L14-L18.

[14] R. C. Tolman, Static solutions of einstein's field equations for spheres of fluid, Physical Review 55 (1939) 364.

[15] J. R. Oppenheimer and G. M. Volkoff, On massive neutron cores, Physical Review 55 (1939) 374.

[16] S. Bonazzola, E. Gourgoulhon, M. Salgado and J. Marck, Axisymmetric rotating relativistic bodies: a new numerical approach for'exact'solutions, Astronomy and Astrophysics $\mathbf{2 7 8}$ (1993) 421-443.

[17] D. Chatterjee, T. Elghozi, J. Novak and M. Oertel, Consistent neutron star models with magnetic field dependent equations of state, Mon. Not. Roy. Astron. Soc. 447 (2015) 3785.

[18] J. L. Noronha and I. A. Shovkovy, Color-flavor locked superconductor in a magnetic field, Physical Review D $\mathbf{7 6}$.

[19] M. Bocquet, S. Bonazzola, E. Gourgoulhon and J. Novak, Rotating neutron star models with magnetic field, Astron. Astrophys. 301 (1995) 757, [gr-qc/9503044].

[20] M. Hempel, V. Dexheimer, S. Schramm and I. Iosilevskiy, Noncongruence of the nuclear liquid-gas and deconfinement phase transitions, Phys. Rev. C 88 (Jul, 2013) 014906. 
[21] P. Papazoglou, D. Zschiesche, S. Schramm, J. Schaffner-Bielich, H. Stoecker et al., Nuclei in a chiral SU(3) model, Phys.Rev. C59 411-427.

[22] V. Dexheimer and S. Schramm, Proto-Neutron and Neutron Stars in a Chiral SU(3) Model, Astrophys.J. 683 943-948.

[23] V. Dexheimer and S. Schramm, A Novel Approach to Model Hybrid Stars, Phys.Rev. C81 045201.

[24] K. Fukushima, Chiral effective model with the Polyakov loop, Phys.Lett. B591 277-284.

[25] S. Weinberg, Why do quarks behave like bare Dirac particles?, Phys.Rev.Lett. 65 1181-1183.

[26] E. J. Ferrer, V. de la Incera, D. M. Paret, A. P. Martínez and A. Sanchez, Insignificance of the anomalous magnetic moment of charged fermions for the equation of state of a magnetized and dense medium, Phys. Rev. D 91 (Apr, 2015) 085041.

[27] S. Chakrabarty, Quark Matter in Strong Magnetic Field, Phys. Rev. D54 (1996) 1306-1316, [hep-ph/9603406].

[28] I.-S. Suh, G. Mathews and F. Weber, Comment on 'Can there be a quark matter core in a magnetar?', Phys.Rev.D (2001), [astro-ph/0110069].

[29] A. Perez Martinez, H. Perez Rojas, H. J. Mosquera Cuesta, M. Boligan and M. G. Orsaria, Quark stars and quantum-magnetically induced collapse, Int. J. Mod. Phys. D14 (2005) 1959, [astro-ph/0506256].

[30] R. G. Felipe, A. P. Martinez, H. P. Rojas and M. Orsaria, Magnetized strange quark matter and magnetized strange quark stars, Phys. Rev. C77 (2008) 015807, [0709.1224].

[31] V. Dexheimer, R. Negreiros and S. Schramm, Hybrid Stars in a Strong Magnetic Field, Eur.Phys.J. A48 189.

[32] V. Dexheimer, R. Negreiros, S. Schramm and M. Hempel, Deconfinement to Quark Matter in Neutron Stars - The Influence of Strong Magnetic Fields, AIP Conf.Proc. 1520 264-269.

[33] M. Strickland, V. Dexheimer and D. P. Menezes, Bulk Properties of a Fermi Gas in a Magnetic Field, Phys. Rev. D86 (2012) 125032.

[34] V. Dexheimer, D. P. Menezes and M. Strickland, The influence of strong magnetic fields on proto-quark stars, J. Phys. G41 (2014) 015203.

[35] A. G. Pili, N. Bucciantini and L. Del Zanna, Axisymmetric equilibrium models for magnetized neutron stars in General Relativity under the Conformally Flat Condition, Mon. Not. Roy. Astron. Soc. 439 (2014) 3541-3563.

[36] J. Frieben and L. Rezzolla, Equilibrium models of relativistic stars with a toroidal magnetic field, Mon. Not. Roy. Astron. Soc. 427 (2012) 3406-3426.

[37] A. Mastrano, A. G. Suvorov and A. Melatos, Neutron star deformation due to poloidal-toroidal magnetic fields of arbitrary multipole order: a new analytic approach, Mon. Not. Roy. Astron. Soc. 447 (2015) 3475. 